\title{
Modeling of variant copies of subunit D1 in the structure of photosystem II from Thermosynechococcus elongatus
}

\author{
Bernhard Loll1,a,b, Matthias Broser',a, Peter \\ B. Kós ${ }^{3}$, Jan Kern ${ }^{2, *}$, Jacek Biesiadka', Imre \\ Vass $^{3}$, Wolfram Saenger ${ }^{1}$ and Athina Zouni ${ }^{2}$ \\ ${ }^{1}$ Institute of Chemistry and Biochemistry/ \\ Crystallography, Free University Berlin, Takustrasse 6, \\ D-14195 Berlin, Germany \\ ${ }^{2}$ Max-Volmer-Laboratories for Biophysical Chemistry, \\ Technical University Berlin, Strasse des 17 Juni 135, \\ D-10623 Berlin, Germany \\ ${ }^{3}$ Institute of Plant Biology, Biological Research Center, \\ H-6726 Szeged, Hungary \\ * Corresponding author \\ e-mail: kern@chem.tu-berlin.de
}

\section{Introduction}

Photosystem II (PSII) is a multi-subunit pigment-protein complex embedded in the thylakoid membrane of cyanobacteria, green algae and higher plants. It captures sunlight by chlorophyll a (Chla) molecules located in the two antenna proteins CP47 and CP43. The excitation energy is transferred to the photochemical reaction center $(\mathrm{RC})$, a heterodimer of proteins D1 and D2 that harbor the primary electron donor (P680) formed by Chla molecule(s). P680 is oxidized to the radical cation $\mathrm{P} 60^{+}$, and the released electron travels along the electron transfer chain (ETC) featuring two pairs of Chla, one pair of pheophytin a (Pheoa), two plastoquinones $\left(Q_{A}\right.$ and $\left.Q_{B}\right)$ and one non-heme $\mathrm{Fe}^{2+}$. $\mathrm{P} 680^{+\bullet}$ is re-reduced via redoxactive Tyr161 $\left(\mathrm{Tyr}_{\mathrm{z}}\right)$ by an electron from the unique $\mathrm{Mn}_{4} \mathrm{Ca}$-cluster, where the oxidation of water to atmospheric oxygen is catalyzed. During water oxidation the $\mathrm{Mn}_{4} \mathrm{Ca}$-cluster cycles through five different oxidation states, which are known as $\mathrm{S}_{\mathrm{i}}$-states $(\mathrm{i}=0-4)$. Recently, new evidence for the existence of additional intermediate states in the reaction cycle was presented (Clausen and Junge, 2004; Haumann et al., 2005). By storing redox equivalents at the $\mathrm{Mn}_{4} \mathrm{Ca}$-cluster, PSII couples the oneelectron photochemistry of the reaction center with the four-electron chemistry of water oxidation. After two cycles, doubly reduced and protonated $Q_{B}$ is released as plastoquinol $\mathrm{Q}_{\mathrm{B}} \mathrm{H}_{2}$ into the plastoquinone pool in the thylakoid membrane (Wydrzynski and Satoh, 2005; Kern and Renger, 2007). The redox equivalents transferred to mobile $Q_{B} H_{2}$ are used in later reactions for the synthesis of NADPH and finally the reduction of $\mathrm{CO}_{2}$ to carbohydrates (Ke, 2001).

The crystal structure of the oxygen-evolving PSII core complex from the thermophilic cyanobacterium Thermosynechococcus elongatus has been published at $3.0 \AA$ resolution (Loll et al., 2005, 2007). PSII is a homodimer, each monomer being composed of 20 different protein subunits harboring 77 cofactors. The cofactors of the ETC are related by a pseudo-two-fold rotation axis normal to the membrane plane and passing through the non-heme $\mathrm{Fe}^{2+}$ [pseudo $\mathrm{C}_{2}\left(\mathrm{Fe}^{2+}\right)$ ], this symmetry being broken by the $\mathrm{Mn}_{4} \mathrm{Ca}$-cluster on the $\mathrm{D} 1$ side and by two differently oriented carotenoids $\left(\mathrm{Car}_{\mathrm{D} 1} / \mathrm{Car}_{\mathrm{D} 2}\right)$ (Loll et al., 2005). Each of the subunits D1 and D2 features five transmembrane $\alpha$-helices (TMHs) $(\mathbf{a}-\mathbf{e})$ arranged in semicircles. The $\mathrm{N}$ - and $\mathrm{C}$-termini of $\mathrm{D} 1$ and the protein segments connecting the TMHs form short, mostly amphipathic $\alpha$-helices located at the cytoplasmic or lumenal surface of the thylakoid membrane. This architecture mirrors the arrangement of the $L$ and $M$ subunits in the well-resolved purple bacteria reaction center (Deisenhofer et al., 1995), as well as the RC domains of PsaA and PsaB in photosystem I (PSI) (Schubert et al., 1998; Jordan et al., 2001). 
In cyanobacteria, the $p s b A$ gene encodes protein D1 in a precursor form containing a carboxy-terminal (C-terminal) extension of 16 amino acids (Nixon et al., 1992). The unprocessed D1 protein is inserted into the thylakoid membrane, associates with other PSII components and is cleaved after D1-Ala344 by C-terminal protease CtpA to yield the mature and 344 amino acids long protein D1 (Anbudurai et al., 1994; Satoh and Yamamoto, 2007). D1 provides the protein scaffold for the majority of cofactors involved in primary electron transfer that are, from the donor to the acceptor side: $\mathrm{Mn}_{4} \mathrm{Ca}$-cluster, $\mathrm{Tyr}_{\mathrm{Z}}$, chlorophyll pair $\left(\mathrm{P}_{\mathrm{D} 1}\right)$, accessory chlorophyll $\left(\mathrm{Chl}_{\mathrm{D} 1}\right)$, pheophytin acceptor $\left(P e_{D 1}\right.$ ), non-heme $\mathrm{Fe}^{2+}$ (coordinated by D1 and D2) and $\mathrm{Q}_{\mathrm{B}}$ (Loll et al., 2005).

Light provides PSII with the necessary energy to drive photosynthesis; however, light energy in excess has to be avoided. Photosynthetic RCs are optimized for the conversion of light energy into chemical energy via electron transfer reactions that are reversible, and the forward reactions of charge-separated states might decrease due to charge recombination reactions. These back reactions not only reduce the photosynthetic efficiency, but they can cause chemical damage, as at high light intensities Chla triplet states can be populated and react with molecular oxygen to form singlet oxygen that leads to oxidative damage of protein and the embedded pigments (Krieger-Liszkay, 2004).

This damage can be avoided in part, as photosynthetic organisms balance energy input and consumption through dissipation of excess energy, and they regulate the energy flow between the two photosystems through state transition, e.g., by adjusting the outer antenna system of PSII and PSI (Allen, 2003). In addition, green algae and higher plants have evolved the xanthophyll cycle, a mechanism for survival under high-light conditions, which is absent in cyanobacteria.

When subunit D1 is damaged by radicals, it is replaced by newly synthesized, intact D1 with high turnover rate relative to other thylakoid proteins (Mattoo et al., 1984; Ohad et al., 1984). This must be a rather complex process, as PSII has to be at least partially disassembled (van Wijk et al., 1997), the freshly synthesized protein D1 has to be assembled into the complex and all cofactors have to be correctly incorporated.

In many cyanobacteria, the $p s b A$ gene coding for subunit D1 occurs in several copies with different nucleotide sequences (Golden, 1995), whereas in higher plants and algae there is only one single copy. The diverse genes for D1 are differentially regulated in response to changes in light intensity (Bustos et al., 1990; Sicora et al., 2006), while in higher plants the turnover of the D1 polypeptide is accompanied by reversible phosphorylation of N-terminal threonines (Mattoo et al., 1989; Allen, 1992; Aro et al., 1993).

The three D1 genes of Synechococcus sp. PCC 7942 are regulated differentially in response to changes in light intensity. In the laboratory, this is demonstrated by a lowto high-light shift. At low light, $>80 \%$ of the $p s b A$ transcripts are from $p s b A l$, and the only D1 protein detectable in the thylakoid membrane is PsbA1. Upon a shift to high light, the $p s b A / l$ and $p s b A I l l$ genes are immediately induced, the $p s b A l$ message being diminished (reviewed in Golden, 1995). Whereas in higher plants and green algae the expression of the chloroplast harbored $p s b A$ gene encoding protein D1 is mainly regulated posttranscriptionally by mRNA stability and translation (Rochaix, 1992), in cyanobacteria the transcription of the psbA genes is modulated by light. The expression of psbAll and psbAIIl in Synechococcus PCC 7942 is proportional to light intensity, whereas that of $p s b A l$ is inversely related to light intensity (Schaefer and Golden, 1989).

Differential regulation of $p s b A$ genes also occurs in $T$. elongatus and leads to the replacement of PsbA1, which is the dominating species under normal growth conditions with PsbA3 under exposure to high light and other conditions of photo-oxidative stress (Kós et al., 2008). Because the PsbA1 and PsbA3 protein isoforms differ in 21 amino acid positions, we have performed structural modeling to clarify the functional role of these amino acid replacements in light acclimation of PSII. Our results show that most of the amino acid changes occur in the direct vicinity to redox-active cofactors. Possible structural and mechanistic consequences for electron transfer are discussed.

\section{Results}

\section{Expression levels of $p s b A$ genes}

In the genome of the thermophilic photoautotrophic cyanobacterium $T$. elongatus, three copies of the $p s b A$ gene have been identified that encode distinct forms of the D1 protein (Nakamura et al., 2002). The regulation of $p s b A$ expression in $T$. elongatus is studied in less detail, but a recent study by Kós et al. (2008) revealed a light and temperature dependence of the mRNA levels of the three $p s b A$ copies. In $T$. elongatus, the main $p s b A$ transcript stems from psbAl under low-light conditions - psbAlll being up-regulated under high-light and low temperature stress.

The distribution of the psbA transcripts under identical cell culture conditions, as described by Kern et al. (2005), was examined (Figure 1). The analysis of the mRNA pool of the three $p s b A$ copies showed an initial decrease of the $p s b A$ I mRNA level to approximately $90 \%$ at lower cell densities (higher light intensity), whereas the mRNA level of $p s b A l l l$ increased to a maximum of $10 \%$ (Figure $1 A, B$ ). This shift was reversed at later stages of the cell culture. In addition, eight different samples of cells in the late logarithmic phase (at this stage cells are normally harvested for protein extraction) were analyzed. The average composition was $98.75( \pm 0.47) \%$ of $p s b A l, 0.61$ $( \pm 0.14) \%$ of $p s b A l l$, as well as $0.64( \pm 0.39) \%$ of $p s b A l l l$, indicating that $p s b A / l$ and $p s b A / I I$ mRNA are only present in trace amounts under the applied culture conditions at high cell density (O.D. ca. 1 a.u.). This confirmed that the prevalent form in the protein samples used for crystallization experiments and subsequent structure determination by X-ray crystallography is PsbA1 (Loll et al., 2005). The observed shift in psbAl and psbAIIl levels can best be explained by the changing light conditions due to the increasing cell density during cell culture. PsbAll was only present in trace amounts under all conditions stud- 

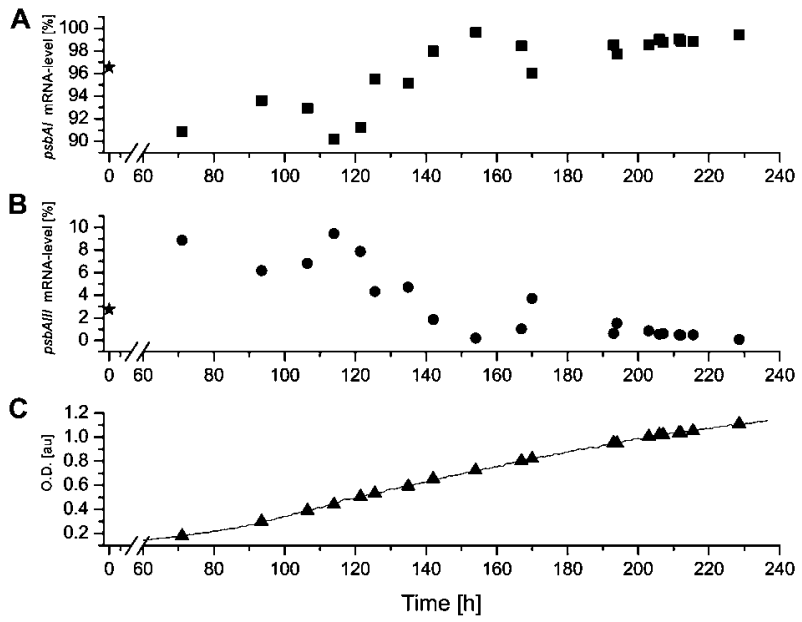

Figure 1 Distribution of the $p s b A$ mRNA pool during cell cultivation.

The $p s b A l$ mRNA level and $p s b A I l l$ mRNA level are related to the total mRNA of all three psbA genes $(=100 \%)$. Samples of cells from three different culture cycles were taken at different times during cultivation; early taken samples had a low cell density and therefore a higher average light intensity per cell than samples taken at higher cell densities. The samples with an O.D. of approximately 1 a.u. were taken from eight different culture cycles. (A) Squares illustrate mRNA level of $p s b A l$ and the asterisk initial inoculation (O.D. ca. 1 a.u. - 1 day stirred at room temperature). The mRNA level of $p s b A l$ increases from $90 \%$ at low O.D. (<0.6 a.u.) to $98.5 \%$ at high O.D. (approximately 1 a.u.). (B) Circles indicate mRNA level of $p s b A$ All and the asterisk initial inoculation. The mRNA level of psbAlll decreases from $10 \%$ at lower O.D. ( $<0.6$ a.u.) to $0.5 \%$ at higher O.D. (approximately 1 a.u.). (C) Representative growth curve (black line) of the T. elongatus cells cultivated in a photobioreactor. Triangles show the times when cells were taken for mRNA analysis. ied and its expression level seems not to respond to environmental conditions, in agreement with a recent study (Kós et al., 2008).

\section{Modeling of D1 copies}

To elucidate the possible function of the various D1 copies (Motoki et al., 1995), we modeled the appropriate substitutions in the $3.0 \AA$ crystal structure, which originally contained the PsbA1 variant of D1. The models were subsequently energy minimized to ensure the optimal geometry of the protein matrix. Even though some of the substituted amino acids had side chains of different mass, they never produced serious steric conflicts, indicating that the substitutions are probably not associated with significant changes in the overall architecture of PSII.

PsbA1 with 360 amino acids differs in 35 residues from PsbA2 and in 21 residues from PsbA3 (Figure 2). The amino acid sequences of PsbA1 and PsbA2 show 90\% identity and $95 \%$ similarity, while for PsbA1 and PsbA3 the sequence identity is $94 \%$ and the similarity $98 \%$, and PsbA2 and PsbA3 show $91 \%$ sequence identity and $98 \%$ similarity. These numbers show that D1 copies are highly conserved. However, the nature and distribution of the mutations strongly suggest an influence on basic functionalities of PSII. If all three copies of protein D1 are compared, the segments with most significant variations are the $\mathrm{N}$-terminus (amino acids 1-30) with 10 variant residues and the segment spanning 15 residues (144-158) upstream of redox-active $\mathrm{Tyr}_{\mathrm{z}}$ on the stromal side of $\mathrm{TMH}-\mathrm{c}$ with six variations (Figure 2). As we only detected significant expression levels for $p s b A l$ and
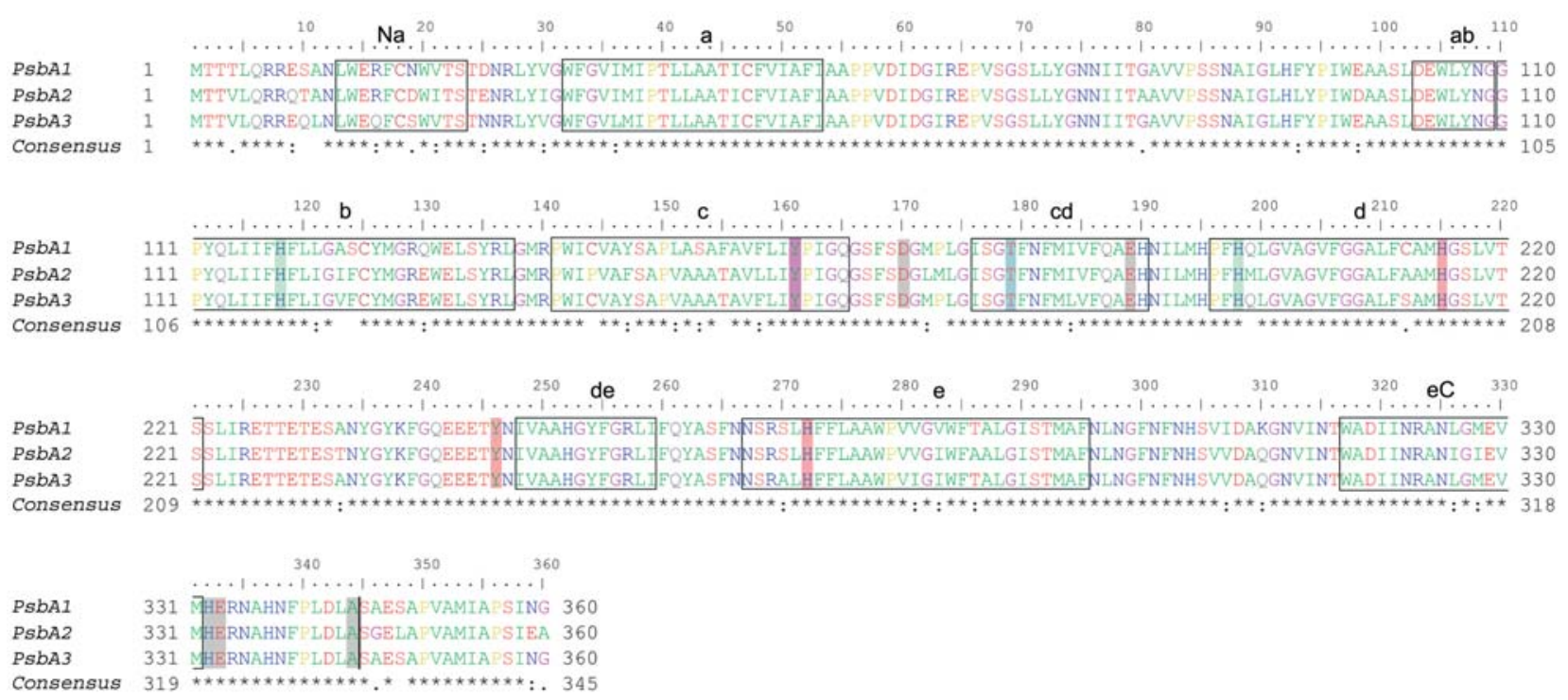

Figure 2 Sequence alignment of the three PsbA (PsbA1, PsbA2, PsbA3) protein copies of T. elongatus.

TMH (a-e) and mostly aliphatic $\alpha$-helices ( $\mathbf{N a}, \mathbf{a b}, \mathbf{c d}$, de and $\mathbf{e C}$ ) are indicated by black rectangles. Amino acids in the consensus sequence are marked as follows: identical by asterisks, similar by two dots, less similar by one dot and not conserved by spacing. Amino acids that are acidic and hydrophilic $(D, E, T, S)$ are colored red, basic residues $(K, R, H)$ blue, apolar residues $(A, F, I, L, M$, V, W) green, Gly purple, Pro yellow, Gln gray. Chla-coordinating histidine residues are marked with green background, other indirectly coordinating residues in D1 (Thr179) are indicated by light-blue background. His215, His272 and Tyr246 coordinating the non-heme iron are marked with red background. Residues coordinating the $\mathrm{Mn}_{4} \mathrm{Ca}$-cluster are indicated by gray background and $\operatorname{Tyr}_{\mathrm{Z}}\left(\operatorname{Tyr}_{161}\right)$ is marked with violet background. The cutting site of protease CtpA is indicated by a vertical black line. 
Table 1 Location of variant residues within the copies of PsbA1 and PsbA3 of T. elongatus.

\begin{tabular}{|c|c|c|c|c|}
\hline $\begin{array}{l}\text { Residue } \\
\text { number }\end{array}$ & PsbA1 & PsbA3 & $\begin{array}{l}\text { Secondary } \\
\text { structure } \\
\text { element }\end{array}$ & Neighboring cofactor \\
\hline 36 & Ile & Leu & TMH-a & $\mathrm{Chlz}_{\mathrm{D} 1}$ \\
\hline 121 & Leu & Ile & TMH-b & $\begin{array}{l}\text { Chlz } z_{D 1} \text { and acyl of DGDGa } 201 \text { and phytol } \\
\text { of Chla41 of CP43 }\end{array}$ \\
\hline 123 & Ala & Val & TMH-b & phytol of $\mathrm{P}_{\mathrm{D} 1}$ and $\mathrm{PheO}_{\mathrm{D} 1}$ \\
\hline 124 & Ser & Phe & TMH-b & $\begin{array}{l}\text { Chla41 of CP43 and acyl of } \\
\text { DGDGa } 201 / 202\end{array}$ \\
\hline 130 & Gln & Glu & TMH-b & $\mathrm{Pheo}_{\mathrm{D} 1} / \mathrm{H}$-bond donor \\
\hline 151 & Leu & Val & TMH-c & acyl of DGDGa 202 \\
\hline 153 & Ser & Ala & TMH-C & phytol of $P_{D 1}$ \\
\hline 155 & Phe & Thr & $\mathrm{TMH}-\mathrm{C}$ & acyl of lipid 202 and quinone cavity \\
\hline 184 & lle & Leu & $\alpha$-helix cd & $P_{D 1}$ \\
\hline 212 & Cys & Ser & $\mathrm{TMH}-\mathrm{d}$ & Non-heme $\mathrm{Fe}^{2+}$ \\
\hline 270 & Ser & Ala & TMH-e & H-bond to head group of SQDQ 204 \\
\hline 281 & Val & Ile & TMH-e & Alkyl of LHGc 203 and quinone cavity \\
\hline 283 & Val & lle & TMH-e & $\mathrm{Pheo}_{\mathrm{D} 1}$ and phytol of $\mathrm{P}_{\mathrm{D} 1}$ \\
\hline 310 & Lys & Gln & loop & Contact to PsbE and PsbV \\
\hline
\end{tabular}

Only the variances close to cofactors are listed.

aDigalactosyldiacylglycerol.

bSulfoquinovosyldiacylglycerol.

'Phosphatidylglycerol.

psbAlll (see above), Table 1 summarizes 14 of the most significant variations between PsbA1 and PsbA3 and provides a short description of their location within the three-dimensional structure of D1 (Figure 3A). Expecting a higher degree of conservation of amino acids in the transmembrane-spanning region than in the loop regions (and in the N-terminus), the variance of this segment is significant, as it might have an influence on the electron and proton transfer properties of $\mathrm{Tyr}_{\mathrm{Z}}, \mathrm{P}_{\mathrm{D} 1}$ and $\mathrm{Pheo}_{\mathrm{D} 1}$ (Table 1). Interestingly, comparing PsbA1 and PsbA3, variant residues are also located on TMH-a (one), -b (four), -d (one) and -e (three) of D1 (Figures 2 and 3). A closer look at the positions of residues within these TMHs shows that some are located close to other redox-active cofactors $\left(\mathrm{P}_{\mathrm{D} 1}, \mathrm{Chlz}_{\mathrm{D} 1}, \mathrm{Pheo}_{\mathrm{D} 1}, \mathrm{Chl}_{\mathrm{D} 2}\right.$, non-heme $\mathrm{Fe}^{2+}$ and lipid molecules; Figure 3) or in the vicinity of Chla41 embedded in CP43, or they even might have an influence on the binding affinity to other subunits (PsbE and PsbV, see Table 1). The protein environment of some cofactors of the RC is not affected by D1 variants. For example, the binding niche of $\mathrm{Car}_{\mathrm{D} 1}$, which is oriented approximately perpendicular to the membrane plane and parallel to $\mathrm{TMH}-\mathbf{a}$, is not affected. In the following section, the most significant examples will be described and discussed in terms of potential function and stability/assembly of the core complex.

\section{Inter-subunit interactions in the four-helix bundle formed by TMHs D1-d, D1-e and D2-d, D2-e}

These TMHs are packed against each other and form a rigid scaffold to incorporate most of the cofactors of the ETC, but they also provide the contact interface between the two subunits (Figure 3A). Towards the cytoplasmic side, TMHs D1-d and D2-d intersect in the height of the non-heme $\mathrm{Fe}^{2+}$. In its immediate vicinity towards the lumenal side, D1-Cys212 and D2-Cys211 residing on TMHs D1-d and D2-d are involved in inter-subunit interaction, but do not form a disulfide bridge. The SH-group of D2-Cys211 and the backbone oxygen of D1-Cys212 form a long hydrogen bond of $3.8 \AA$. Interestingly, D1Cys212 is replaced by Ser in PsbA3 that might influence the interactions between D1 and D2 and alter assembly/ disassembly of the PSII complex. In a recent study, it was demonstrated that modifications in this region result in changes of the local flexibility of the reaction center and seem to function as modulator in controlling the activation entropy of $Q_{A}{ }^{-}$to $Q_{B}$ electron transfer (Shlyk-Kerner et al., 2006).

\section{Inter-subunit interactions - D1 and CP43}

Six of the variant residues (residues 121, 123, 124, 151, 153 and 281) are located towards CP43 in the middle of the membrane plane. D1-Leu121, D1-Ala123 and D1Ser124 residing on TMH D1-b are in vicinity of Chla41 of CP43, D1-Leu151 and D1-Ser153 residing on $\mathrm{TMH}$ D1-c, the helix facing TMH CP43-f, and D1-Val281 at TMH D1-e. Most of the connections between these residues and CP43 are bridged by cofactors, such as Chla41, as well as lipids at the interface of D1 and CP43. Because all these residues are located in the middle of the membrane plane and on neighboring TMHs, one can assume an influence of this concerted modification on the D1 exchange, during which the CP43 subunit has to be detached and rebound (Rokka et al., 2005).

\section{Interaction of D1, cyt $b-559$ and cyt $c-550$}

The variant residue D1-Lys310 in PsbA1 (Figure 2 and Table 1) is located on a loop at the lumenal side and at a strategic position in close vicinity to PsbE ( $\alpha$-chain of cyt $b$-559) and the membrane-extrinsic PsbV (cyt c-550). The exchange of D1-Lys310 in PsbA1 to Gln in PsbA3 may lead to an inter-subunit hydrogen bond between D1-GIn310 and the backbone oxygen of PsbE-GIn58, the side chain of which forms a hydrogen bond to PsbV-Glu28. 
A

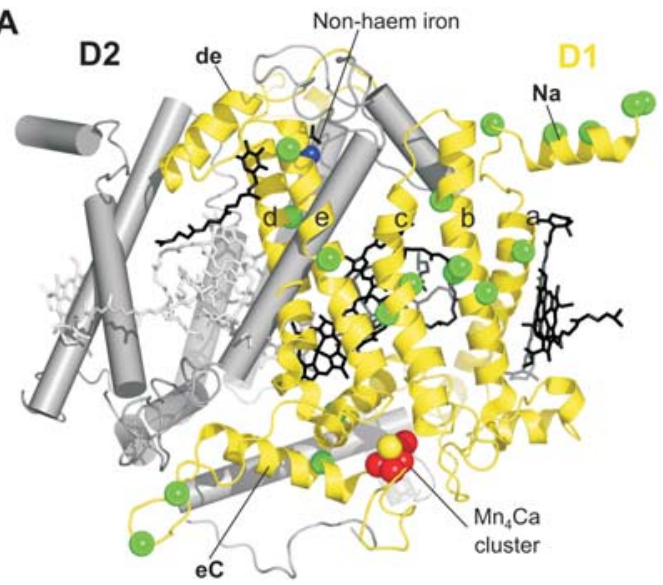

B

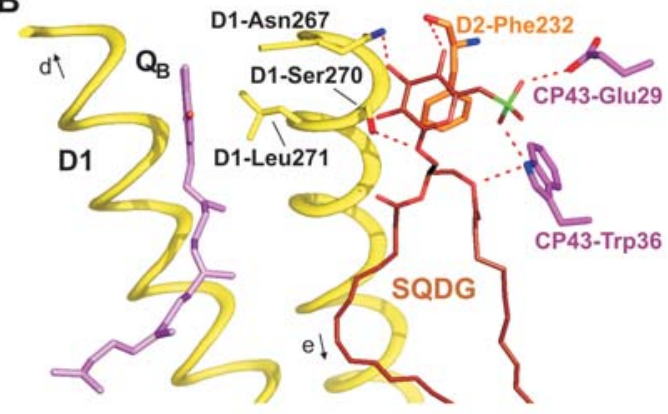

C

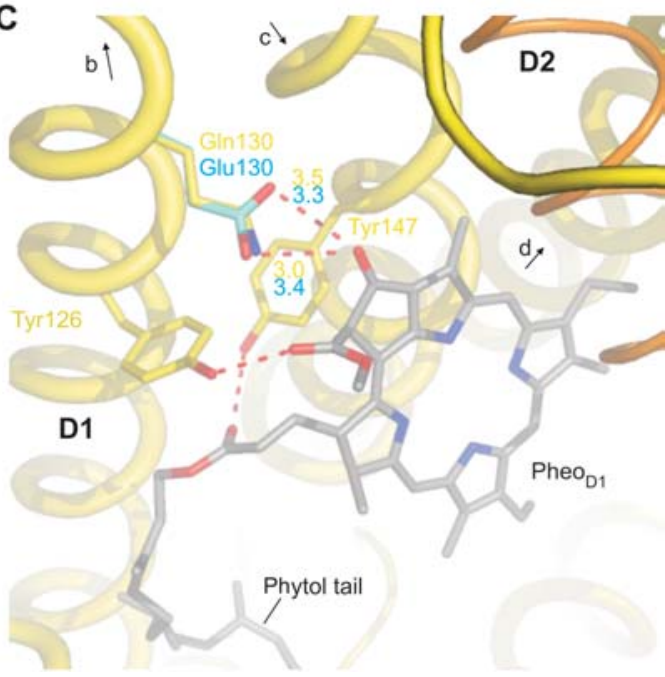

Modulated protein-cofactor interactions (cofactordirected interactions) and secondary quinone $\left(Q_{B}\right)$

Thermoluminescence analyses to study the charge recombination events between the acceptor and donor side of Synechococcus sp. PCC 7942 indicated that in stressed cells exhibiting the high-light form (PsbA2 in the case of Synechococcus sp.), the redox potential of $Q_{B}$ became lower approaching that of $Q_{A}$ (Sane et al., 2002), and this in turn would open the possibility for $P 680^{+} Q_{A}{ }^{-}$ recombination. The accumulation of reduced $Q_{A}\left(Q_{A}{ }^{-}\right)$has been shown to inhibit the formation of radical pair $\mathrm{P}^{2} 60^{+} \mathrm{Pheo}$, and thus promoting the triplet formation of $P 680$ (Vass et al., 1992). A change in the $Q_{B}$ redox potential could be associated with major changes found at the $\mathrm{N}$-terminal $\alpha$-helix ( $\mathrm{Na}$ in Figures 2 and $3 \mathrm{~A}$ ) of $\mathrm{D} 1$ in the high-light form compared to the low-light form (Golden
Figure 3 Variant residues within the PsbA3 copy of subunit D1. (A) View along the membrane plane of reaction center proteins D1 (yellow) and D2 (gray). All other protein subunits and cofactors within the PSII complex are omitted for clarity. Variant residues within PsbA1 and PsbA3 are depicted by green balls. The cofactors coordinated by D1 are drawn in black, whereas cofactors coordinated by D2 are in gray. The cations of the $\mathrm{Mn}_{4} \mathrm{Ca}-$ cluster are drawn as spheres in red $(\mathrm{Mn})$ and yellow $\left(\mathrm{Ca}^{2+}\right)$, as well as the non-heme $\mathrm{Fe}^{2+}$ (blue). Cofactors and lipid molecules surrounding the $\mathrm{RC}$ subunits have been omitted for clarity. (B) SQDG (brown) is embedded at the subunit interface of D1PsbA1 (yellow), D2 (orange) and CP43 (magenta). The $\mathrm{Q}_{\mathrm{B}}$ molecule is drawn in violet. Only amino acid residues involved in hydrogen bonding with the SQDG lipid are indicated by red dashed lines. The replacement of Ser270 to Ala270 changes the hydrogen bonding network in the SQDG binding pocket. (C) Binding pocket of $\mathrm{PheO}_{\mathrm{D} 1}$. The D1 protein is shown in yellow and D2 in orange. Hydrogen bonds are indicated by red dashed lines, $\mathrm{Pheo}_{\mathrm{D} 1}$ and hydrogen bonding amino acids in stick presentation (yellow for PsbA1 and cyan for PsbA3), nitrogen blue and oxygen red. Distances are given in Ångstrom units.

et al., 1986) that could exert unspecific long-range effects on the $Q_{B}$ binding pocket and consequently modulate the redox properties of $Q_{B}$.

Analysis of the decay of fluorescence yield after single flash illumination in $T$. elongatus shows differences between high-light (PsbA3) and low-light (PsbA1) form of D1, mainly in the middle phase (Kós et al., 2008), which is assigned to re-oxidation of $Q_{A}$ by quinone molecules moving into the $Q_{B}$ binding site after flash excitation. This difference may be caused by an altered $Q_{B}$ binding site or changes in the quinone diffusion pathway between the two D1 forms. Therefore, the exchange of D1-Ser270 in PsbA1 to Ala in PsbA3 might be significant as the hydroxyl group of serine provides a hydrogen-bond to the oxygen of the glycerol function of the sulfoquinovosyldiacylglycerol (SQDG) which is close to the $Q_{B}$ site (Figure 3B). In the PsbA3 copy, the alanine at this position will alter the hydrogen-bonding network associated with the head group of SQDG and might change the specificity for lipid binding. In the direct neighborhood, D1-Leu271 points into the $Q_{B}$ pocket, and therefore it is feasible to assume that changes in the lipid environment or lipid specificity could influence the plastoquinone exchange through the proposed lipid diffusion pathway (Loll et al., 2005, 2007).

\section{Pheo $_{\mathrm{D} 1}$ binding pocket}

Amino acid sequence analysis reveals that higher plants exclusively have glutamate at position D1-130, whereas cyanobacteria have either glutamate or glutamine. Only the genes psbAl of $T$. elongatus, psbAl of Synechococcus sp. WH 8102, psbA of Prochlorococcus marinus subsp. pastoris str. CCMP1378, psbAl, psbAll, and psbAlll of Synechocystis sp. PCC 6803, as well as psbA and psbAl of Nostoc sp. PCC 7120 and Anacystis nidulans have glutamine at this position.

Position D1-130 possibly modulates the hydrogen bonding pattern and charge distribution around $\mathrm{PheO}_{\mathrm{D} 1}$, as D1-Gln130 in PsbA1 is changed to Glu in PsbA3 (Table 1 and Figure $3 \mathrm{C}$ ). Spectroscopic studies on a Synechocystis mutant with exchange from Gln to Glu at 
position D1-130 revealed a shift of the absorption maximum of the $Q_{x}$ absorption band in the Pheo $_{D 1}$ spectrum (Giorgi et al., 1996). It was demonstrated that the variant $\mathrm{E} 130 \mathrm{Q}$ down-shifts the $\mathrm{E}_{\mathrm{M}}$ of $\mathrm{Pheo}_{\mathrm{D} 1}$ by $33 \mathrm{mV}$ in Synechocystis (Merry et al., 1998) and by $38 \mathrm{mV}$ as determined by TL measurements (Cser and Vass, 2007), as well as by $70 \mathrm{mV}$ in Chlamydomonas reinhardti (Cuni et al., 2004). This change in redox potential should increase the free energy gap between the long lived $S_{2} Q_{A}{ }^{-}$and $\mathrm{S}_{2} \mathrm{Pheo}^{-}$state and therefore slow down the indirect recombination process going through the charge-separated state $\mathrm{P} 60^{+} \mathrm{Pheo}$. A weakening or loss of the hydrogen bond to $\mathrm{Pheo}_{\mathrm{D} 1}$ is expected to destabilize Pheo $_{\mathrm{D} 1}{ }^{-}$, thereby rendering its redox potential more negative. This was shown in Synechocystis by measurements of the equilibrium constant between the excited singlet state $\mathrm{P} 60^{*}$ and the $\mathrm{P} 680^{+} \mathrm{Pheo}^{-}$radical pair state and of the quantum yield for nanosecond stabilization of the radical pair (Giorgi et al., 1996; Merry et al., 1998).

Measurements of the recombination rate of $S_{2} Q_{A}{ }^{-}$in the presence of either PsbA1 or PsbA3 in T. elongatus show a much smaller difference between both forms compared to the difference between wild-type Synechocystis 6803 and a D1-GIn130Glu variant (Kós et al., 2008). Therefore, the authors discussed that a parallel change from PsbA1 to PsbA3 might compensate at least partly the effect of the single D1-GIn130Glu exchange in T. elongatus. Candidates for this compensating effect are exchanges of D1-Ser124 to Phe and D1-Leu151 to Val (Table 1). In our structural model, these residues are located towards CP43, but we did not observe any direct influence, i.e., no change in the hydrogen bond of D1-Tyr126 with respect to the binding pocket of $\mathrm{PheO}_{\mathrm{D} 1}$.

In the vicinity of D1-Ser124, the relatively flexible hydrophobic environment provides enough space for the bulky phenylalanine which might replace some water molecules not observed at the current resolution. The benzene ring of the phenylalanine in PsbA3 is very close to Chla41 of CP43 (in van der Waals contact with one of the methyl groups and $4 \AA$ away from the conjugated $\pi$-system of Chla), but the influence on the exitonically coupled Chla molecules in CP43 seems to be negligible.

\section{$P_{D 1}$ binding pocket}

According to recent studies, P680 probably consists of the Chla pair $\mathrm{P}_{\mathrm{D} 1 / \mathrm{D} 2}$ and/or the two accessory Chla $\mathrm{Chl}_{\mathrm{D} 1 / \mathrm{D} 2}$ (Groot et al., 2005). This would allow these cofactors to act as a collective state, thereby promoting fast and efficient charge separation (Peterman et al., 1998; Barter et al., 2003). Furthermore, $\mathrm{Chl}_{\mathrm{D} 1}$ was suggested to be the primary donor in charge separation (Dekker and Van Grondelle, 2000; Prokhorenko and Holzwarth, 2000). The hydrophobic binding pocket of $P_{D 1}$ (Table 1 ) is affected by changes in amino acids 123,153 , 184 and 283 that will modulate the properties of the bound Chla molecule.

A closer look into the binding pocket of $P_{D 1}$ reveals that the hydrophilic residue D1-Ser153 in the PsbA1 copy is replaced by hydrophobic side chain of Ala in the PsbA3 copy. As this residue is located in close vicinity to the carbonyl functions of the chlorin moiety, it might be worthwhile to speculate that it might coordinate a water molecule when the PsbA1 copy is present, which mediates a hydrogen bond to $P_{D 1}$ and thereby influences its properties.

\section{Lipid binding pockets}

The polar lipid head groups are coordinated in PSII by specific and mainly hydrogen bonding interactions close to the stromal and lumenal sides of the subunits and by a larger number of non-polar interactions between the hydrophobic lipid tails and the proteins (Loll et al., 2007). Changes in the binding pockets of lipid head groups might lower their affinity to PSII and may possibly result in binding of lipid molecules with altered head groups. Several amino acid changes either close to the head groups or to the acyl chains of lipids (Table 1) were observed for the D1 copies.

\section{Discussion}

Cyanobacteria developed a unique protection mechanism to avoid photo-inhibition, which employs differential regulation of $p s b A$ genes in response to changes in environmental light conditions. The occurrence of alternative $p s b A$ gene copies coding for different variants of subunit D1 is, to our knowledge, only limited to this group of photosynthetic organisms. However, cyanobacteria are not uniform with respect to this, and the number of $p s b A$ copies varies substantially between their species from one in Prochlorococcus marinus to six in Anabaena variabilis. The number of two to three copies represents the most typical situation, occurring in the best studied strains of Synechococcus and Synechocystis. The number of $p s b A$ genes appears to be correlated with the overall complexity of the genome, when size and total number of encoded polypeptides are considered. Similar rules apply to the number of $p s b D$ genes coding for subunit D2. The numbers of $p s b D$ copies are systematically lower compared to $p s b A$ and do not exceed two (in A. variabilis).

To be sure that $T$. elongatus PSII used for crystallization shows a minimal heterogeneity regarding the D1 protein, we analyzed the mRNA levels of the psbA genes under the culturing conditions used to obtain crystallizationgrade PSII. This analysis revealed that our preparations exclusively contain the PsbA1 form. Recent results by Kós et al. (2008) showed a light intensity and temperature depending shift between the PsbA1 and PsbA3 forms and that the associated exchange of amino acids affects the electron transport characteristics of PSII in T. elongatus. A similar trend for the shift between PsbA1 and PsbA3 depending on light intensity was also observed in our present study.

The residues which are prone to exchange between different PsbA copies are in general highly conserved among all known D1 sequences, and amino acid exchanges at these positions in the various copies of cyanobacterial PsbA proteins are highly conserved. Nevertheless, the pattern of combination of exchanges varies between copies and species. A change in the hydrophobicity of the binding pockets of the six central chlorin cofactors of the ETC occurs commonly upon change 
from one PsbA copy to another. This change in hydrophobicity is found for side chains at positions 130 and 153. In PsbA1 of $T$. elongatus, all of these residues are hydrophilic and/or form hydrogen bonds to one of the embedded cofactors, whereas in PsbA3 two hydrophilic interactions are lost. For other cyanobacteria with several copies of the psbA gene, e.g., A. variabilis, Nostoc or Synechocystis, changes in the hydrophobicity of residues in the binding pockets of the central ETC cofactors can be predicted based on their amino acid sequences, but the pattern of substitutions is different from species to species. For example, in $A$. variabilis one copy shows four hydrophilic residues, whereas in the other five copies three of them are replaced by hydrophobic residues. These dramatic changes might modulate the redox properties of the cofactors within the D1 branch and could have an influence on electron transfer rates under different environmental conditions.

The highly variable $\mathrm{N}$-terminus of D1 is not involved in any cofactor binding. However, very recent data demonstrate that the first 20-25 amino acid residues at the $\mathrm{N}$-terminus are involved in the degradation of photodamaged D1 protein via interaction with the $\mathrm{FtsH}$ protease (Komenda et al., 2007). Previous data obtained with Synechocystis PCC 6803 mutants, which express either the D1:1 (low-light) or D1:2 (high-light) isoform of Synechococcus PCC 7942, showed that increased phototolerance of D1:2 containing PSII arises partly from enhanced repair capacity and partly from decreased extent of photo-damage (Tichy et al., 2003). Thus, modification of the $\mathrm{N}$-terminal region of $\mathrm{PsbA3}$ relative to PsbA1 in $T$. elongatus might play a role in more efficient D1 degradation, which is a prerequisite of PSII repair.

The model described here for the PsbA3 copy of subunit D1 elucidates details of the unique structural and functional features of this reaction center subunit. It provides specific details about protein-protein and proteinpigment interactions and provides an insight into possible mechanistic aspects. This model will serve as a sound basis for mutagenetic studies that further explore the variant amino acid residues and shed light on the translation of the different copies of psbA genes depending on environmental conditions.

\section{Materials and methods}

\section{RT-PCR analysis}

RNA was isolated by the hot phenol method from frozen cell pellets as described earlier (Kós et al., 2008), with minor modifications. The isolated RNA was precipitated with $\mathrm{LiCl}$ and subsequently treated with Turbo DNA-free (Ambion, Austin, TX, USA) to remove traces of genomic DNA. RNA $(2 \mu \mathrm{g})$ was reverse-transcribed using H-MuLV (Fermentas, Vilnius, Lithuania). In the quantitative PCR reaction (Q-PCR), aliquots of the resulted CDNA were used as template. Q-PCR was carried out on an ABI 7000 Sequence Detection System (Applied Biosystems Inc., Foster City, CA, USA) using Power-SYBR green PCR Master-mix from the same manufacturer.

\section{Cell culture}

Cyanobacteria were grown in a photobioreactor (PBR25 IGV Potsdam, Germany, and Sartorius-BBI Systems, Melsungen,
Germany) as described previously (Kern et al., 2005) in modified Castenholz Medium D $\left(\mathrm{CaCl}_{2}\right.$ and Fe-EDTA instead of $\mathrm{CaSO}_{4}$ and $\mathrm{FeCl}_{3}$ ) at $50^{\circ} \mathrm{C}$. The $\mathrm{pH}$ was kept constantly at $\mathrm{pH} 7.8$ by automatically adding $\mathrm{CO}_{2}$, and samples were taken at different time points during cultivation. Light intensity was measured in front of and behind the glass tube $(40 \mathrm{~mm}$ thick) relative to the light source using an LI-250 light meter (LI-COR Inc., Lincoln, NE, USA).

\section{Coordinates and modeling}

The atomic coordinates of the $3.0 \AA$ PSII crystal structure are deposited in the Protein Data Bank under accession code 2AXT (Loll et al., 2005). DNA sequences of the different D1 genes were taken from CYANOBASE (Nakamura et al., 2002) and translated to the corresponding protein sequences. Variant residues of D1 copies (Figure 2) were modeled with the program ' $\mathrm{O}$ ' (Jones et al., 1991). Generation of hydrogen atom positions and energy minimization were performed using the CNS program package (Brünger et al., 1998), and the CCP4 package (Collaborative Computing Project 4, 1994) was used to manipulate atomic coordinate files. Final coordinates of the models were evaluated with PROCHECK (Laskowski et al., 1993). The amino acid alignment was performed with program ClustalW (Thompson et al., 1994) and adjusted manually. Figures were prepared with PYMOL (http://www.pymol.org).

\section{Acknowledgments}

The authors are grateful to the Deutsche Forschungsgemeinschaft, Sonderforschungsbereich 498 projects A4 and C7 (to B.L., M.B., J.K., J.B., A.Z.), to the Fonds der Chemischen Industrie (to W.S.) and to the Max-Planck Society (to B.L.) for financial support. P.K. and I.V. are grateful to the European Union, FP6 program (STREP-SOLAR-H-516510) for financial support. We also would like to thank $A$. Wilde and D. Dienst for initial support in mRNA isolation.

\section{References}

Allen, J.F. (1992). Protein phosphorylation in regulation of photosynthesis. Biochim. Biophys. Acta 1098, 275-335.

Allen, J.F. (2003). Botany. State transitions - a question of balance. Science 299, 1530-1532.

Anbudurai, P.R., Mor, T.S., Ohad, I., Shestakov, S.V., and Pakrasi, H.B. (1994). The ctpA gene encodes the C-terminal processing protease for the D1 protein of the photosystem II reaction center complex. Proc. Natl. Acad. Sci. USA 91, 8082-8086.

Aro, E.M., Virgin, I., and Andersson, B. (1993). Photoinhibition of photosystem-2 - inactivation, protein damage and turnover. Biochim. Biophys. Acta 1143, 113-134.

Barter, L.M., Durrant, J.R., and Klug, D.R. (2003). A quantitative structure-function relationship for the photosystem II reaction center: supermolecular behavior in natural photosynthesis. Proc. Natl. Acad. Sci. USA 100, 946-951.

Brünger, A.T., Adams, P.D., Clore, G.M., DeLano, W.L., Gros, P., Grosse-Kunstleve, R.W., Jiang, J.S., Kuszewski, J., Nilges, M., Pannu, N.S., et al. (1998). Crystallography and NMR system: a new software suite for macromolecular structure determination. Acta Crystallogr. D 54, 905-921.

Bustos, S.A., Schaefer, M.R., and Golden, S.S. (1990). Different and rapid responses of four cyanobacterial psbA transcripts to changes in light intensity. J. Bacteriol. 172, 1998-2004.

Clausen, J. and Junge, W. (2004). Detection of an intermediate of photosynthetic water oxidation. Nature $430,480-483$. 
Collaborative Computing Project 4. (1994). The CCP4 suite programs for protein crystallography. Acta Crystallogr. D D50, 760-763.

Cser, K. and Vass, I. (2007). Radiative and non-radiative charge recombination pathways in photosystem II studied by thermoluminescence and chlorophyll fluorescence in the cyanobacterium Synechocystis 6803. Biochim. Biophys. Acta 1767, 233-243.

Cuni, A., Xiong, L., Sayre, R., Rappaport, F., and Lavergne, J. (2004). Modification of the pheophytin midpoint potential in photosystem II: modulation of the quantum yield of charge separation and of charge recombination pathways. Phys. Chem. Chem. Phys. 6, 4825-4831.

Deisenhofer, J., Epp, O., Sinning, I., and Michel, H. (1995). Crystallographic refinement at $2.3 \AA$ resolution and refined model of the photosynthetic reaction center from Rhodopseudomonas viridis. J. Mol. Biol. 246, 429-457.

Dekker, J.P. and Van Grondelle, R. (2000). Primary charge separation in photosystem II. Photosynth. Res. 63, 195-208.

Giorgi, L.B., Nixon, P.J., Merry, S.A., Joseph, D.M., Durrant, J.R., De Las Rivas, J., Barber, J., Porter, G., and Klug, D.R. (1996). Comparison of primary charge separation in the photosystem II reaction center complex isolated from wild-type and D1-130 mutants of the cyanobacterium Synechocystis PCC 6803. J. Biol. Chem. 271, 2093-2101.

Golden, S.S. (1995). Light-responsive gene expression in cyanobacteria. J. Bacteriol. 177, 1651-1654.

Golden, S.S., Brusslan, J., and Haselkorn, R. (1986). Expression of a family of $p s b A$ genes encoding a photosystem II polypeptide in the cyanobacterium Anacystis nidulans R2. EMBO J. 5, 2789-2798.

Groot, M.L., Pawlowicz, N.P., van Wilderen, L.J.G.W., Breton, J., van Stokkum, I.H., and van Grondelle, R. (2005). Initial electron donor and acceptor in isolated photosystem II reaction centers identified with femtosecond mid-IR spectroscopy. Proc. Natl. Acad. Sci. USA 132, 13087-13092.

Haumann, M., Liebsch, P., Muller, C., Barra, M., Grabolle, M., and Dau, $\mathrm{H}$. (2005). Photosynthetic $\mathrm{O}_{2}$ formation tracked by time-resolved X-ray experiments. Science 310, 1019-1021.

Jones, T.A., Zou, J.Y., Cowan, S.W., and Kjeldgaard, M. (1991). Improved methods for binding protein models in electron density maps and the location of errors in these models. Acta Crystallogr. A 47, 110-119.

Jordan, P., Fromme, P., Witt, H.T., Klukas, O., Saenger, W., and Krauss, N. (2001). Three-dimensional structure of cyanobacterial photosystem I at 2.5 A resolution. Nature 411, 909-917.

$\mathrm{Ke}$, B. (2001). Photosynthesis - Photobiochemistry and Photobiophysics, Vol. 10 (Dordrecht, The Netherlands: Kluwer Academic Publishers).

Kern, J., Loll, B., Lüneberg, C., DiFiore, D., Biesiadka, J., Irrgang, K.-D., and Zouni, A. (2005). Purification, characterisation and crystallisation of photosystem II from Thermosynechococcus elongatus cultivated in a new type of photobioreactor. Biochim. Biophys. Acta 1706, 147-157.

Kern, J. and Renger, G. (2007). Photosystem II: structure and mechanism of the water:plastoquinone oxidoreductase. Photosynth. Res. 94, 183-202.

Komenda, J., Tichy, M., Prasil, O., Knoppova, J., Kuvikova, S., de Vries, R., and Nixon, P.J. (2007). The exposed N-terminal tail of the D1 subunit is required for rapid D1 degradation during photosystem II repair in Synechocystis sp. PCC 6803. Plant Cell 19, 2839-2854.

Kós, P.B., Deák, Z., Cheregi, O., and Vass, I. (2008). Differential regulation of $p s b A$ and $p s b D$ gene expression, and the role of the different D1 protein copies in the cyanobacterium Thermosynechococcus elongatus BP-1. Biochim. Biophys. Acta 1777, 74-83.

Krieger-Liszkay, A. (2004). Singlet oxygen production in photosynthesis. J. Exp. Bot. 56, 337-346.
Laskowski, R.A., McArthur, M.W., Moss, D.S., and Thornton, J.M. (1993). PROCHECK: a program to check the stereochemical quality of protein structures. J. Appl. Crystallogr. 26, 283-291.

Loll, B., Kern, J., Saenger, W., Zouni, A., and Biesiadka, J. (2005). Towards complete cofactor arrangement in the $3.0 \AA$ resolution structure of photosystem II. Nature 438, 10401044.

Loll, B., Kern, J., Saenger, W., Zouni, A., and Biesiadka, J. (2007). Lipids in photosystem II: interactions with protein and cofactors. Biochim. Biophys. Acta 1767, 509-519.

Mattoo, A.K., Hoffman-Falk, H., Marder, J.B., and Edelman, M. (1984). Regulation of protein metabolism: coupling of photosynthetic electron transport to in vivo degradation of the rapidly metabolized 32-kilodalton protein of the chloroplast membranes. Proc. Natl. Acad. Sci. USA 81, 1380-1384.

Mattoo, A.K., Marder, J.B., and Edelman, M. (1989). Dynamics of the photosystem II reaction center. Cell 56, 241-246.

Merry, S.A.P., Nixon, P.J., Barter, L.M.C., Schilstra, M., Porter, G., Barber, J., Durrant, J.R., and Klug, D.R. (1998). Modulation of quantum yield of primary radical pair formation in photosystem II by site-directed mutagenesis affecting radical cations and anions. Biochemistry 37, 17439-17447.

Motoki, A., Shimazu, T., Hirano, M., and Katoh, S. (1995). The two psbA genes from the thermophilic cyanobacterium Synechococcus elongatus. Plant Physiol. 108, 1305-1306.

Nakamura, Y., Kaneko, T., Sato, S., Ikeuchi, M., Katoh, H., Sasamoto, S., Watanabe, A., Iriguchi, M., Kawashima, K., Kimura, T., et al. (2002). Complete genome structure of the thermophilic cyanobacterium Thermosynechococcus elongatus BP-1. DNA Res. 9, 123-130.

Nixon, P.J., Trost, J.T., and Diner, B.A. (1992). Role of the carboxy terminus of polypeptide D1 in the assembly of a functional water-oxidizing manganese cluster in photosystem II of the cyanobacterium Synechocystis sp. PCC 6803: assembly requires a free carboxyl group at C-terminal position 344. Biochemistry 31, 10859-10871.

Ohad, I., Kyle, D.E., and Arntzen, C.J. (1984). Membrane protein damage and repair: removal and replacement of inactivated 32-kilodalton polypeptides in chloroplast membranes. J. Cell Biol. 99, 481-485.

Peterman, E.J.G., van Amerongen, H., van Grondelle, R., and Dekker, J.P. (1998). The nature of the excited state of the reaction center of photosystem II of green plants: a highresolution fluorescence spectroscopy study. Proc. Natl. Acad. Sci. USA 95, 6128-6133.

Prokhorenko, V.I. and Holzwarth, A.R. (2000). Primary processes and structure of photosystem II reaction center: a photon echo study. J. Phys. Chem. 104, 11563-11578.

Rochaix, J.D. (1992). Post-transcriptional steps in the expression of chloroplast genes. Annu. Rev. Cell Biol. 8, 1-28.

Rokka, A., Suorsa, M., Saleem, A., Battchikova, N., and Aro, E.M. (2005). Synthesis and assembly of thylakoid protein complexes: multiple assembly steps of photosystem II. Biochem. J. 388, 159-168.

Sane, P.V., Ivanov, A.G., Sveshnikov, D., Huner, N.P., and Oquist, G. (2002). A transient exchange of the photosystem II reaction center protein D1:1 with D1:2 during low temperature stress of Synechococcus sp. PCC 7942 in the light lowers the redox potential of $Q_{B}$. J. Biol. Chem. 277, 32739-32745.

Satoh, K. and Yamamoto, Y. (2007). The carboxyl-terminal processing of precursor D1 protein of the photosystem II reaction center. Photosynth. Res. 94, 203-215.

Schaefer, M.R. and Golden, S.S. (1989). Differential expression of members of a cyanobacterial psbA gene family in response to light. J. Bacteriol. 171, 3973-3981.

Schubert, W.D., Klukas, O., Saenger, W., Witt, H.T., Fromme, P., and Krauss, N. (1998). A common ancestor for oxygenic and anoxygenic photosynthetic systems: a comparison based on 
the structural model of photosystem I. J. Mol. Biol. 280, 297-314.

Shlyk-Kerner, O., Samish, I., Kaftan, D., Holland, N., Sai, P.S., Kless, H., and Scherz, A. (2006). Protein flexibility acclimatizes photosynthetic energy conversion to the ambient temperature. Nature 442, 827-830.

Sicora, C., Appleton, S.E., Brown, C.M., Chung, J., Chandler, J., Cockshutt, A.M., Vass, I., and Campbell, D.A. (2006). Cyanobacterial $p s b A$ families in Anabaena and Synechocystis encode trace, constitutive and UVB-induced D1 isoforms. Biochim. Biophys. Acta 1757, 47-56.

Thompson, J.D., Higgins, D.G., and Gibson, T.J. (1994). CLUSTAL W: improving the sensitivity of progressive multiple sequence alignment through sequence weighting, positionspecific gap penalties and weight matrix choice. Nucleic Acids Res. 22, 4673-4680.

Tichy, M., Lupínková, L., Sicora, C., Vass, I., Kuvikova, S., Prasil, O., and Komenda, J. (2003). Synechocystis 6803 mutants expressing distinct forms of the Photosystem II D1 protein from Synechococcus 7942: relationship between the $p s b A$ coding region and sensitivity to visible and UV-B radiation. Biochim. Biophys. Acta 1605, 55-66.

van Wijk, K.J., Roobol-Boza, M., Kettunen, R., Andersson, B., and Aro, E.M. (1997). Synthesis and assembly of the D1 protein into photosystem II: processing of the C-terminus and identification of the initial assembly partners and complexes during photosystem II repair. Biochemistry 36, 6178-6186.

Vass, I., Styring, S., Hundal, T., Koivuniemi, A., Aro, E., and Andersson, B. (1992). Reversible and irreversible intermediates during photoinhibition of photosystem II: stable reduced $\mathrm{Q}_{\mathrm{A}}$ species promote chlorophyll triplet formation. Proc. Natl. Acad. Sci. USA 89, 1408-1412.

Wydrzynski, T. and Satoh, K. (2005). Photosystem II: the lightdriven water:plastoquinone oxidoreductase. In: Advances in Photosynthesis and Respiration Series, Vol. 22 (Dordrecht, The Netherlands: Springer).

Received December 2, 2007; accepted January 16, 2008 